\title{
A GWAS-identified susceptibility locus on chromosome 11q13.3 and its putative molecular target for prediction of postoperative prognosis of human renal cell carcinoma
}

\author{
TONG SU $^{1 *}$, YIFANG HAN $^{1 *}$, YONGWEI YU ${ }^{2 *}$, XIAOJIE TAN ${ }^{1}$, XIAOPAN LI ${ }^{1}$, JIANGUO HOU $^{3}$, YAN DU $^{1}$, \\ JIAN SHEN ${ }^{3}$, GUOPING WANG ${ }^{1}$, LIYE MA ${ }^{4}$, SHUANG JIANG ${ }^{5}$, HONGWEI ZHANG ${ }^{1}$ and GUANGWEN CAO ${ }^{1}$ \\ ${ }^{1}$ Department of Epidemiology, Second Military Medical University; Departments of ${ }^{2}$ Pathology, \\ ${ }^{3}$ Urology and ${ }^{4}$ General Surgery, and ${ }^{5}$ Physical Examination Center, Changhai Hospital, \\ Second Military Medical University, Shanghai 200433, P.R. China
}

Received January 21, 2013; Accepted June 19, 2013

DOI: 10.3892/ol.2013.1422

\begin{abstract}
Genome-wide association studies have been used to identify single nucleotide polymorphisms (SNPs) associated with renal cell carcinoma (RCC) in European individuals. The current study aimed to evaluate the correlation between significant SNPs identified in European individuals and the occurrence and postoperative prognosis of RCC in Chinese individuals. A total of 400 cases and 806 controls were involved in the current study. rs4765623, rs7105934, rs7579899 and rs1867785 were genotyped using qPCR, and the expression of cyclin D1 in renal tissue and RCCs was determined via western blotting and immunohistochemistry. The correlation between the SNPs/cyclin D1 expression and overall survival was evaluated using multivariate Cox regression analyses. Of the four SNPs, only rs7105934 was found to significantly correlate with RCC risk in Chinese individuals. The rs7105934 $\mathrm{GA}+\mathrm{AA}$ genotype was correlated with a reduced risk of RCC with an odds ratio of 0.64 (95\% confidence interval [CI], 0.43-0.96), following adjustment for age. This genotype was found to independently predict an improved postoperative prognosis in the multivariate analysis, with a hazard ratio (HR) of 0.12 (95\% CI, 0.02-0.93). Expression of cyclin D1, a putative regulated protein of rs7105934, did not vary in adjacent renal tissue and tumors when compared with that of various rs7105934 genotypes. However, cyclin D1 expression in RCCs inversely correlated with advanced tumor stage, and moderate to high expression of cyclin D1 in RCCs independently
\end{abstract}

Correspondence to: Professor Guangwen Cao, Department of Epidemiology, Second Military Medical University, 800 Xiangyin Road, Shanghai 200433, P.R. China

E-mail: gcao@smmu.edu.cn

*Contributed equally

Key words: single nucleotide polymorphism, renal cell carcinoma, risk, prognosis, cyclin D1 predicted improved postoperative prognosis, with an HR of 0.13 (95\% CI, 0.02-0.96). Observations of the present study indicate that the rs7105934 A allele is associated with reduced risk and improved postoperative prognosis of RCC; however, this effect is unlikely to be caused by cyclin D1 expression.

\section{Introduction}

Renal cell carcinoma (RCC) accounts for $80-90 \%$ of cases of kidney cancer. The incidence rate of RCC is generally higher in more developed countries than in less developed countries (1). The rate of RCC is 2-fold higher in males than in females and increases with age. RCC is a pathogenically heterogeneous disease and clear cell RCC (ccRCC) accounts for $70-80 \%$ of cases of kidney cancer. Epidemiological studies have identified that hypertension, obesity, cigarette smoking and exposure to trichloroethylene are environmental risk factors of RCC; however, the impact of these factors may vary among populations (2-4). Environmental factors and their interaction with genetic factors are hypothesized to affect the risk of RCC $(5,6)$. Single nucleotide polymorphisms (SNPs) in cancer growth-related cytokines, including vascular endothelial growth factor, Dickkopf-3 in the Wnt pathway and blood pressure genes, are associated with RCC risk (7-9). SNPs of metabolic enzymes, including cytochrome P450 mono-oxygenases, $\mathrm{N}$-acetyltransferase and glutathione $\mathrm{S}$-transferase, are associated with RCC risk in Caucasians; although, these correlations have not been replicated in Chinese individuals $(4,10)$, indicating that the association between genetic predisposition and RCC risk alters among various populations. However, a number of studies using candidate-gene approaches have not yielded a conclusive result (4-10).

Surgical resection remains the curative therapy for RCC patients; although, patients treated surgically have 5- and 10-year relative survival rates of 72 and 63\%, respectively (11). Previous studies have shown that specific clinical and molecular factors have predictive values for postoperative prognosis: Advanced tumor stages; circulating molecules, including C-reactive protein and erythrocyte polyamines; molecules in the tumor, including L1 cell adhesion molecules; and chromosomal 
variations, including $10 \mathrm{q}$ and $13 \mathrm{q}$ deletions, and D9S168 microsatellite alterations. These factors are capable of predicting a poor postoperative prognosis for RCC (12-16). Genetic predispositions, including the Dickkopf-2 rs17037102 polymorphism, interleukin-4 haplotype -589T-33T, MDM2 rs2279744 polymorphism and miRNA-related genetic polymorphisms are closely associated with RCC clinical outcome (8,17-19).

A previous genome-wide association study (GWAS) in RCC cases and controls of European background revealed that two loci mapped to EPAS1, encoding hypoxia inducible factor (HIF)-2 $\alpha$, on 2p21 (rs11894252 and rs7579899), a locus on 11q13.3 (rs7105934) and a locus mapped to SCARB1, encoding the scavenger receptor class $B$, member 1 , on $12 \mathrm{q} 24.31$ (rs4765623) were significantly associated with RCC susceptibility (20). rs7105934 at 11q13.3 was detected to modulate the binding and function of HIF-2 $\alpha$ at a transcriptional enhancer of CCND1 (encoding cyclin D1) (21). In the present study, the correlations between SNPs identified in the present GWAS and RCC susceptibility were validated, and the role of SNPs in the postoperative prognosis of RCC was investigated in a Chinese population.

\section{Materials and methods}

Study subjects. A total of 400 pathologically confirmed, sporadic RCC patients diagnosed between November 1998 and November 2011 at the Department of Urology (Changhai Hospital, Second Military Medical University, Shanghai, China) were involved in the present study. In addition, 806 controls that received comprehensive physical examinations, including type-B ultrasonic and blood tests, and were confirmed to be healthy at the Physical Examination Center of Changhai Hospital between 2006 and 2011, were also involved. Individuals who did not have notable metastasis at the time of surgery and did not receive adjuvant therapy after surgery were followed up. Follow-up was initiated 6 months after surgery, performed by telephone or interviews in person at the Outpatient Department every 3 months, in accordance with standard epidemiological procedures. The median follow-up period was 34.0 months (range, 3.0-90.9 months). All participants were of Chinese ethnic origin, and the study protocol conformed to the ethical guidelines of the 1975 Declaration of Helsinki and was approved by the Institutional Review Board of the Second Military Medical University (Shanghai, China). All participants provided written informed consent.

Genotyping of genetic polymorphisms. Genomic DNA was extracted from blood samples using QIAquick PCR purification kits (Qiagen, Hilden, Germany). rs4765623, rs7105934, rs7579899 and rs1867785 (highly correlated with rs11894252) were genotyped using fluorescent-probe qPCR in a LightCycler $^{\mathrm{TM}} 480$ (Roche Diagnostics, Mannheim, Germany). Primers and probes (TaqMan or Minor Groove Binder) were designed and synthesized by GeneCore BioTechnologies Co., Ltd. (Shanghai, China), and the sequences of the primers and probes are shown in Table I. Each reaction mixture contained $0.2 \mu \mathrm{mol} / 1$ primers and probes, as well as 0.1-0.5 $\mu \mathrm{g}$ purified templates in the Premix Ex Taq ${ }^{\mathrm{TM}}$ reaction system (Takara Bio, Inc., Shiga, Japan). Three duplicative samples were run with a template-free control.
Western blotting. Protein was extracted from the adjacent renal tissue of fresh RCC specimens and quantified using cell lysis buffer and Enhanced BCA Protein Assay kits (Beyotime Institute of Biotechnology, Jiangsu, China). Extracted proteins were resolved by routine PAGE gels and transferred onto polyvinylidene difluoride membranes. The membranes were incubated overnight with rabbit monoclonal antibodies against cyclin D1 (1:1,000; Epitomics Inc., Burlingame, CA, USA) and $\beta$-actin (1:1,000; Cell Signaling Technology, Inc., Danvers, MA, USA), washed and incubated with HRP-conjugated anti-rabbit or anti-mouse IgG (Cell Signaling Technology, Inc.) for $2 \mathrm{~h}$. Peroxidase activity was developed by ECL detection reagent (GE Healthcare, Amersham, UK) according to the manufacturer's instructions. The signal intensity of each band was quantified using Genetools software, version 4.02 (Syngene, Cambridge, UK). The relative intensity of each sample was calculated as the intensity of the cyclin D1 band (raw value-background noise)/the intensity of the $\beta$-actin band (raw value-background noise).

Immunohistochemistry. Antibodies against cyclin D1 (1:100) were used for immunohistochemistry of RCC specimens in accordance with methods previously described for western blotting (22). Sections were independently assessed by four researchers who were blinded to the clinical information. Immunostaining intensity was scored as follows: Negative (-), $<5 \%$ positive cancer cells; weak $(+), 5-20 \%$ positive; moderate $(++), 20-60 \%$ positive; and strong $(+++),>60 \%$ positive. There was $>95 \%$ agreement among the researchers and disagreements were resolved by consensus. A total of 90 paraffin-embedded samples were used in immunohistochemical assay.

Statistical analysis. Differences in categorical variables were evaluated using the $\chi^{2}$ test. Hardy-Weinberg equilibrium (HWE) was examined online (http://ihg.gsf.de/ihg/snps.html) and linkage disequilibrium (LD) analyses for the SNPs involved in the current study and those identified in previous GWAS $(23,24)$ were performed using online Haploview 4.2 software (http://hapmap.ncbi.nlm.nih.gov). Differences in the relative intensities of the western blotting bands among the samples of various genotypes were analyzed using the Student's t-test. Unconditional logistic regression analysis was used to obtain an odds ratio (OR) for each SNP with RCC and a 95\% confidence interval (CI). Overall survival was analyzed using the Kaplan-Meier method and the log-rank test was used to compare survival curves. Forward stepwise multivariate Cox regression analysis $\left(\mathrm{P}_{\text {entry }}=0.05\right.$ and $\mathrm{P}_{\text {removal }}=0.10$ ) was performed to determine the factors contributing independently to RCC prognosis. The correlation between immunostaining scores for cyclin D1 and RCC stage and the rs7105934 genotypes was determined by Spearman's rank correlation analysis, respectively. All statistical tests were two-sided and conducted using SPSS for Windows, version 16.0 (SPSS, Inc., Chicago, IL, USA). P<0.05 was considered to indicate a statistically significant difference.

\section{Results}

Correlation between the four SNPs and RCC risk. Table II shows the characteristics of the RCC patients and healthy 
Table I. Primers and probes for genotyping the four single nucleotide polymorphisms.

\begin{tabular}{|c|c|c|c|}
\hline & Variation & Primers 5'-3' & Probes \\
\hline rs7105934 & $\mathrm{G} / \mathrm{A}$ & $\begin{array}{l}\text { GAGGAATGATGAACAAACTGTGGTA } \\
\text { CAGAACATCACATAAATGGAATCATACA }\end{array}$ & $\begin{array}{l}\text { FAM-CCAAAATGCATCGTGCTAAGAAGCC-TAMRA } \\
\text { HEX-TCCAAAATGCATCATGCTAAGAAGCC-TAMRA }\end{array}$ \\
\hline rs1867785 & $\mathrm{A} / \mathrm{G}$ & $\begin{array}{l}\text { GGACTTCTCTCTCССТTCACCCT } \\
\text { TCCTGTGTTTCCAAGAGTTCTCAGA }\end{array}$ & $\begin{array}{l}\text { FAM-AAATTAGCTTCGTTGACCTCAGCCAGC-TAMRA } \\
\text { HEX-ATTAGCTTCGTCGACCTCAGCCAGC-TAMRA }\end{array}$ \\
\hline rs7579899 & $\mathrm{A} / \mathrm{G}$ & $\begin{array}{l}\text { ACACAGCCAAATCCAAGTCAGA } \\
\text { TGACCAAACACTAGGAAAGGAGAAG }\end{array}$ & $\begin{array}{l}\text { FAM-ACACCCTGTACAAAGCACTGCGACC-TAMRA } \\
\text { HEX-ACACCCTGTACAGAGCACTGCGACC-TAMRA }\end{array}$ \\
\hline rs 4765623 & $\mathrm{C} / \mathrm{T}$ & $\begin{array}{l}\text { GGTCTCGCGCATGTGTCA } \\
\text { CCAGATGCGTTCAGCAGTTC }\end{array}$ & $\begin{array}{l}\text { FAM- AGTACAGCCACCTCGGAGAGCCACT-TAMRA } \\
\text { HEX- AGTACAGCCACCTTGGAGAGCCACTG-TAMRA }\end{array}$ \\
\hline
\end{tabular}

Table II. Demographic and clinicopathological characteristics of the study subjects.

\begin{tabular}{lccc}
\hline Characteristics & Cases, $\mathrm{n}(\%)$ & Controls, n (\%) & P-value \\
\hline Age, years & & & \\
$\leq 40$ & $31(7.8)$ & $36(4.5)$ & 0.008 \\
$40-60$ & $210(52.5)$ & $380(47.1)$ & \\
$60-80$ & $147(36.8)$ & $352(43.7)$ & \\
$>80$ & $12(3.0)$ & $38(4.7)$ & \\
Gender & & & \\
Male & $274(68.5)$ & $548(68.0)$ & \\
Female & $126(31.5)$ & $258(32.0)$ & \\
Histology & & & \\
Clear cell & $373(93.3)$ & - & \\
Papillary & $12(3.0)$ & - & \\
Chromophobe & $8(2.0)$ & - & \\
Unclassified & $7(1.8)$ & - & \\
AJCC (2002) stage & & - & \\
I & $324(81.0)$ & - & \\
II & $33(8.3)$ & $43(10.8)$ & - \\
III & & &
\end{tabular}

AJCC, American Joint Committee on Cancer.

controls from the present study. The gender distribution was not significantly different between the RCC patients and healthy controls; however, the healthy controls were significantly older than the RCC patients (median \pm standard deviation; $60.1 \pm 12.8$ vs. $57.1 \pm 12.8$ years; $\mathrm{P}<0.001)$. The genotyping call rate was $100 \%$ for each SNP, and in the healthy controls, rs4765623, rs7105934, rs7579899 and rs1867785 conformed to HWE $(\mathrm{P}=0.481,0.891,0.352$ and 0.373 , respectively). LD analysis indicated that rs7579899 was markedly correlated with $\mathrm{rs} 1867785\left(\mathrm{r}^{2}=0.988\right.$ in this study population), whereas rs1867785 was not in LD with rs12617313 or rs9679290 ( $\mathrm{r}^{2}<0.05$ in the HapMap Chinese population), the SNPs identified on chromosome 2 p21 by a previous GWAS (24). Table III shows the correlation between the SNPs and RCC risk. Of the four SNPs, rs7105934 only was found to significantly correlate with RCC risk. The dominant model or A allele at rs7105934 was significantly correlated with a reduced risk of RCC following adjustments for age and gender, with a power of $77.4 \%$. As ccRCC is the major histological type, the correlation between the four SNPs and ccRCC risk was examined, and rs7105934 only was found to correlate with the risk of ccRCC. Compared with the GG genotype, the GA + AA genotype of rs7105934 was significantly correlated with a reduced ccRCC risk with an OR of 0.60 (95\% CI, 0.40-0.92; P=0.018), following adjustments for age and gender. By contrast, the A allele of rs7105934 was inversely associated with ccRCC risk following adjustments (OR, 0.62; 95\% CI, 0.41-0.92; $\mathrm{P}=0.018$ ).

Correlation between SNPs and RCC prognosis. Of the RCC patients, 194/400 completed the follow-up and were included in the survival analysis. It was identified that rs7105934 GA + AA genotype was significantly correlated with an improved postoperative prognosis of RCC ( $\mathrm{P}=0.046$; Kaplan-Meier and log-rank test; Fig. 1A). Table IV presents the results of stepwise multivariate Cox regression analysis, indicating that patients with the rs7105934 GA + AA genotype have a decreased risk of mortality (HR, 0.12; 95\% CI, 0.016-0.93; $\mathrm{P}=0.042)$. In addition, advanced tumor stage was also found to independently predict poor survival rates in RCC.

Correlation between rs7105934 genotypes and cyclin D1 expression. Expression of cyclin D1 protein in the adjacent renal tissue of fresh RCC specimens was examined using western blotting. No significant differences were identified in the relative intensity of cyclin D1 protein between patients with the GG genotype and those with the GA + AA genotype of rs7105934 ( $\mathrm{P}=0.514$; Fig. 2A). Cyclin D1 expression in the tumors was examined using immunohistochemistry (Fig. 2B). Immunostaining intensities of cyclin D1 in the cancer cells of the RCC patient specimens did not correlate significantly among the three genotype groups of $r 57105934(\mathrm{P}=0.844)$ or the $\mathrm{GG}$ and GA + AA genotypes of rs7105934 ( $\mathrm{P}=0.884)$. However, immunostaining intensities of cyclin D1 in the cancer cells was inversely correlated with advanced tumor stage $(\mathrm{P}=0.046$; Spearman's correlation coefficient, -0.211; Table V).

Correlation between cyclin D1 expression in cancer cells and $R C C$ prognosis. The association between cyclin D1 expression in cancer cells and RCC prognosis was first evaluated using 
Table III. Correlation between the four SNPs and risk of RCC following adjustment for age and gender.

\begin{tabular}{|c|c|c|c|c|}
\hline Genotype & $\begin{array}{l}\text { Cases, } \\
\mathrm{n}(\%)\end{array}$ & $\begin{array}{c}\text { Controls, } \\
\text { n (\%) }\end{array}$ & $\begin{array}{c}\text { OR } \\
(95 \% \mathrm{CI})\end{array}$ & $\mathrm{P}$-value ${ }^{\mathrm{a}}$ \\
\hline \multicolumn{5}{|l|}{ rs7105934 } \\
\hline GG & $364(91.0)$ & $700(86.8)$ & 1.00 (reference) & \\
\hline GA & $35(8.8)$ & 102 (12.7) & $0.65(0.43-0.97)$ & 0.036 \\
\hline AA & $1(0.3)$ & $4(0.5)$ & $0.47(0.05-4.25)$ & 0.500 \\
\hline GG & $364(91.0)$ & 700 (86.8) & 1.00 (reference) & \\
\hline $\mathrm{GA}+\mathrm{AA}$ & $36(9.0)$ & $106(13.2)$ & $0.64(0.43-0.96)$ & 0.029 \\
\hline $\mathrm{G}$ allele & 763 (95.4) & $1502(93.2)$ & 1.00 (reference) & \\
\hline A allele & 37 (4.6) & $110(6.8)$ & $0.65(0.44-0.95)$ & 0.028 \\
\hline \multicolumn{5}{|l|}{ rs1867785 } \\
\hline AA & $288(72.0)$ & $561(69.6)$ & 1.00 (reference) & \\
\hline AG & $105(26.3)$ & 227 (28.2) & $0.90(0.69-1.19)$ & 0.472 \\
\hline GG & $7(1.8)$ & $18(2.2)$ & $0.76(0.31-1.86)$ & 0.552 \\
\hline AA & $288(72.0)$ & $561(69.6)$ & 1.00 (reference) & \\
\hline $\mathrm{AG}+\mathrm{GG}$ & $112(28.0)$ & 245 (30.4) & $0.89(0.69-1.17)$ & 0.411 \\
\hline A allele & $681(85.1)$ & 1349 (83.7) & 1.00 (reference) & \\
\hline $\mathrm{G}$ allele & 119 (14.9) & $263(16.3)$ & $0.90(0.71-1.14)$ & 0.381 \\
\hline \multicolumn{5}{|l|}{ rs7579899 } \\
\hline AA & 287 (71.8) & $560(69.5)$ & 1.00 (reference) & \\
\hline $\mathrm{AG}$ & $106(26.5)$ & $228(28.3)$ & $0.91(0.69-1.19)$ & 0.495 \\
\hline GG & $7(1.8)$ & $18(2.2)$ & $0.76(0.31-1.86)$ & 0.554 \\
\hline AA & $287(71.8)$ & $560(69.5)$ & 1.00 (reference) & \\
\hline $\mathrm{AG}+\mathrm{GG}$ & $113(28.3)$ & $246(30.5)$ & $0.90(0.69-1.17)$ & 0.432 \\
\hline A allele & $680(85.0)$ & 1348 (83.6) & 1.00 (reference) & \\
\hline $\mathrm{G}$ allele & $120(15.0)$ & 264 (16.4) & $0.90(0.71-1.14)$ & 0.399 \\
\hline \multicolumn{5}{|l|}{ rs4765623 } \\
\hline $\mathrm{CC}$ & $136(34.0)$ & $268(33.3)$ & 1.00 (reference) & \\
\hline $\mathrm{CT}$ & $188(47.0)$ & 385 (47.8) & $0.97(0.74-1.28)$ & 0.845 \\
\hline TT & $76(19.0)$ & $153(19.0)$ & $0.97(0.68-1.37)$ & 0.844 \\
\hline $\mathrm{CC}$ & $136(34.0)$ & $268(33.3)$ & 1.00 (reference) & \\
\hline $\mathrm{CT}+\mathrm{TT}$ & $264(66.0)$ & 538 (66.7) & $0.97(0.75-1.25)$ & 0.822 \\
\hline $\mathrm{C}$ allele & $460(57.5)$ & $921(57.1)$ & 1.00 (reference) & \\
\hline $\mathrm{T}$ allele & $340(42.5)$ & 691 (42.9) & $0.98(0.83-1.17)$ & 0.826 \\
\hline
\end{tabular}

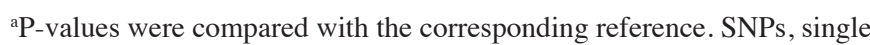
nucleotide polymorphisms; RCC, renal cell carcinoma; OR, odds ratio.

Kaplan-Meier analysis. Moderate to high expression (++/+++) of cyclin D1 in cancer cells predicted an improved postoperative prognosis of RCC when compared with that of negative or weak expression (-/+) $(\mathrm{P}=0.015$; Kaplan-Meier analysis and the log-rank test; Fig. 1B). Multivariate Cox regression analysis indicated that moderate and high expression $(++/+++)$ of cyclin D1 in cancer cells independently predicted an improved postoperative prognosis (HR, 0.13; 95\% CI, 0.02-0.96; $\mathrm{P}=0.045$ ).

\section{Discussion}

In the present study, the correlation between four SNPs identified in a previous GWAS study of a European population and the development and prognosis of RCC in a Chinese popula-
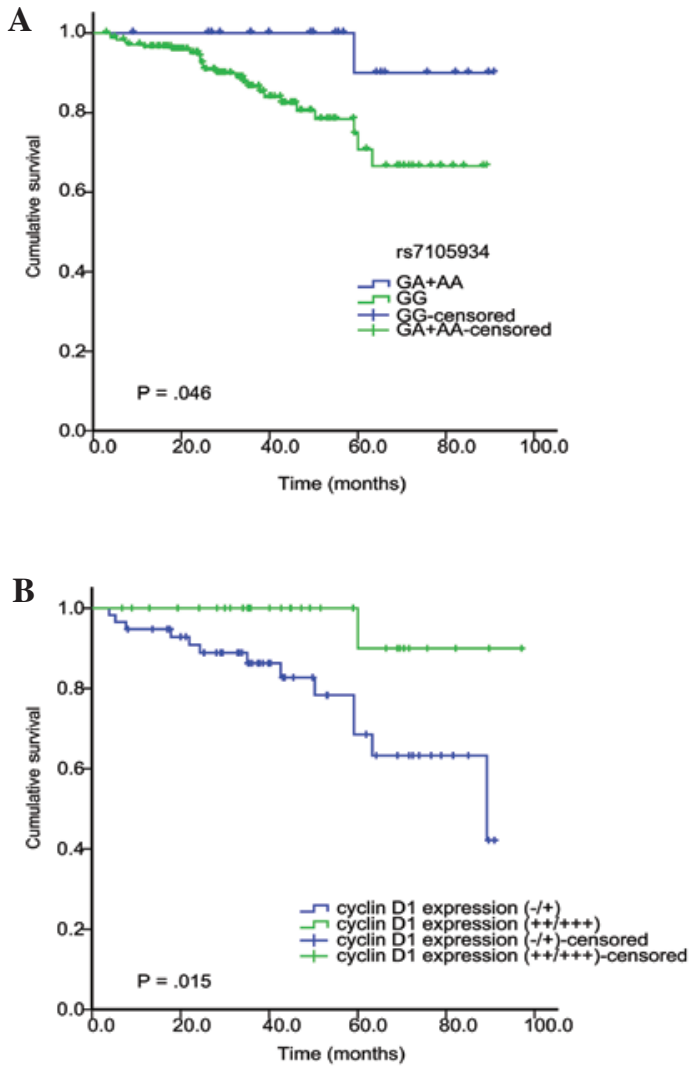

Figure 1. Correlation between rs7105934 or cyclin D1 protein expression in tumor tissues and overall survival of RCC patients following curative nephrectomy (Kaplan-Meier analysis and log-rank test). (A) rs7105934 and overall survival $(\mathrm{n}=194)$. (B) Cyclin D1 protein expression in tumor tissues and overall survival $(\mathrm{n}=90)$. -, negative $(<5 \%$ positive cancer cells); + , weak (5-20\% positive); ++ , moderate (20-60\% positive); +++ , strong ( $>60 \%$ positive); RCC, renal cell carcinoma.

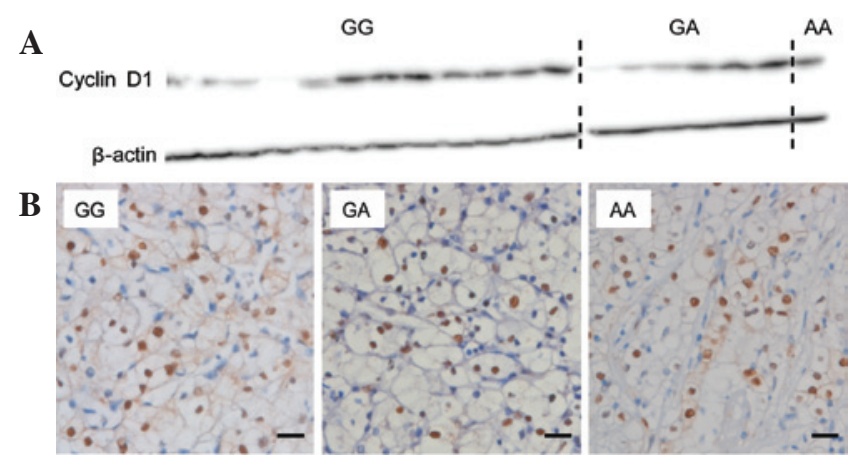

Figure 2. Correlation between rs7105934 genotypes and the expression of cyclin D1 in adjacent renal and RCC tissues. (A) Western blotting to determine cyclin D1 expression in adjacent renal tissue. (B) Immunohistochemistry for the analysis of cyclin D1 expression in RCC tissue. Bar indicates $20 \mu \mathrm{m}$ and brown indicates positive immunostaining. RCC, renal cell carcinoma.

tion was investigated. In the current study, the healthy controls and RCC patients were matched by gender, but the healthy controls were significantly older than the RCC patients. This age contrast may have prevented misclassification bias, as RCC incidence increases with age (2). The current study identified that, of the four SNPs validated, only rs7105934 significantly correlated with the risk of RCC, particularly ccRCC, in the Chinese population. An additional study investigating the 
Table IV. Factors independently associated with overall survival of RCC patients in stepwise multivariate Cox regression analysis.

\begin{tabular}{lccc}
\hline & Cases evaluated, $n$ & Cases of RCC mortality, $n$ & HR (95\% CI) \\
\hline rs7105934 & 172 & 25 & 1.00 (reference) \\
GG & 22 & 1 & $0.12(0.016-0.93)$ \\
GA + AA & & & 0.042 \\
AJCC (2002) stage & 147 & 7 & $1.00($ (reference) \\
I & 12 & 3 & $5.79(1.47-22.82)$ \\
II & 35 & 16 & $15.72(6.36-38.84)$ \\
III & 35 & 0.012 \\
\hline
\end{tabular}

RCC, renal cell carcinoma; HR, hazard ratio; AJCC, American Joint Committee on Cancer.

Table V. Correlation between rs7105934 genotypes or AJCC stage and cyclin D1 expression in ccRCC specimens.

\begin{tabular}{lrrrrr}
\hline & \multicolumn{2}{c}{ Cyclin D1 expression } & & \\
\cline { 2 - 3 } & - & + & ++ ++++ & P-value & $\mathrm{r}_{\mathrm{s}}{ }^{\mathrm{a}}$ \\
\hline rs7105934 & 24 & 25 & 27 & 0.844 & 0.021 \\
GG & 5 & 3 & 5 & & \\
GA & 0 & 0 & 1 & & \\
AA & 24 & 25 & 27 & 0.884 & 0.016 \\
GG & 5 & 3 & 6 & & \\
GA + AA & & & & & \\
AJCC (2002) stage & 16 & 15 & 26 & 0.046 & -0.211 \\
I & 3 & 3 & 2 & & \\
II & 10 & 10 & 5 & & \\
III & & & & & \\
\hline
\end{tabular}

${ }^{\text {aS }}$ pearman's correlation coefficient. AJCC, American Joint Committee on Cancer; -, negative ( $<5 \%$ positive cancer cells); + , weak (5-20\% positive); ++ , moderate ( $20-60 \%$ positive); +++ , strong ( $>60 \%$ positive); ccRCC, clear cell renal cell carcinoma.

association between rs7579899, rs7105934 and rs4765623 and RCC risk in an additional group of Chinese individuals has been reported (25). Similar to the results of the current study, the study identified that only rs7105934 of the genotyped SNPs correlated with a reduced risk of RCC. Therefore, genetic predisposition of RCC appears to differ between the two ethnic groups and rs7105934 may confer genetic susceptibility to RCC in a number of populations.

An important observation of the present study was that the rs7105934 GA + AA genotype is an independent factor for improved RCC prognosis, with an HR of 0.12 (95\% CI, 0.02-0.93), in contrast to advanced tumor stage. The genetic risk factor of RCC development may also modulate RCC outcome. rs7105934 is situated at a transcriptional enhancer $\sim 220-\mathrm{kb}$ telomeric to the cyclin D1 encoding gene, CCND1 (20). SNPs 5-kb centromeric to rs7105934 modulate the binding and function of HIF-2 $\alpha$ at the enhancer for CCND1. The minor (RCC-protective) allele at 11q13.3 disrupts HIF binding, DNA accessibility and interaction with the transcriptional appa- ratus at the $C C N D 1$ enhancer, and alters the allelic balance of CCND1 gene expression (21). The rs7105934 GA genotype appears to be associated with lower levels of CCND1 mRNA in adjacent renal tissue when compared with that of the GG genotype (25). Therefore, the correlation between the GA + AA genotype and an improved prognosis of RCC may be caused by low expression of cyclin D1 in tumors. Of note, the present study identified that moderate to high expression of cyclin D1 is an independent predictor of an improved postoperative prognosis in RCC. No significant differences were identified in cyclin D1 protein expression between specimens with the GG genotype and those with the GA + AA genotype in adjacent renal tissue and in the tumor tissue. In addition, the intensity of cyclin D1 immunostaining did not correlate with the rs7105934 genotype, and these results were not consistent with the hypothesized involvement of rs7105934 genotypes in cyclin D1 expression in RCCs. Thus, cyclin D1 and the rs7105934 SNP may both be significant in the carcinogenesis and postoperative prognosis of RCC, but function independently. Further studies are required to validate and investigate the correlation between rs7105934 genotypes and the expression of cyclin D1 and other candidate target proteins in RCCs.

In the present study, cyclin D1 expression in RCC cells was confirmed to inversely correlate with advanced stages of RCC and represent an independent predictor of improved prognosis of RCC. The results of the current study are similar to those of previous studies reporting that high cyclin D1 expression significantly correlates with an improved prognosis, while low expression of cyclin D1 correlates with a poor prognosis of ccRCC (26-28). The expression of cyclin D1 is markedly correlated with that of p27 in ccRCC; low p27 expression independently predicts a poor prognosis of RCC, whilst low cyclin D1 expression appears to shorten the survival rate of RCC patients (29). These differences may reflect variations in the cyclin D1-related cell biology of various cancer types. Cyclin D1 is an oncoprotein that activates the $\mathrm{G}_{1}-\mathrm{S}$ transition of the cell cycle and functions as a downstream effector of $\beta$-catenin signaling. Downregulation of $\beta$-catenin expression may contribute to the malignant character of RCC and result in tumor progression (30). Cyclin D1 is upregulated in primary ccRCCs, which may contribute to cell proliferation in primary RCCs, and its importance may diminish at later stages of RCC progression due to specific complex mechanisms, including epithelial-mesenchymal transition. 
Several limitations of the present study must be noted: i) follow-up information was only available for 194 study subjects and the majority of individuals without follow-up information were from other provinces of China, which made regular contact difficult; ii) patients were excluded in cases where postoperative therapy was administered, including interferon- $\alpha$ and interleukin-2 treatments, during the follow-up period; iii) epidemiological studies of specific risk factors, including smoking and alcohol consumption, were incomplete and thus not included in the analysis, resulting in loss of data; and iv) SNPs were validated from only one GWAS study (20) and novel RCC-related SNPs on 12p11.23 and 2p21 $(23,24)$ require validation, as these SNPs are not in LD with the four SNPs validated for the present study.

Overall, the present study showed that, of the four RCC-related SNPs identified in a European population, only rs7105934 correlated with RCC risk in a Chinese population. The GA + AA genotype was significantly correlated with reduced risk and an improved postoperative prognosis of RCC. Therefore,rs7105934 may confer genetic susceptibility to RCC in extended populations. No significant differences were identified in the levels of cyclin D1 expression in adjacent renal tissue and RCC cells between patients with the rs7105934 GG genotype and those with the GA + AA genotype. Cyclin D1 expression in RCC cells was inversely correlated with advanced stages of RCC. In addition, moderate to high expression of cyclin D1 was an independent predictor of improved postoperative prognosis of RCC. However, the results do not support the hypothesis that rs7105934 genotypes are involved in the expression of cyclin D1 in the kidneys. Cyclin D1 and the rs7105934 SNP may represent significant factors in RCC, however, their effects appear to be independent. Although validation of these observations is required in independent populations, the current study highlights a novel genetic marker that is capable of predicting the postoperative prognosis of RCC.

\section{Acknowledgements}

The authors would like to thank Guoping Wang, Liye Ma and Shuang Jiang for their assistance with sample collection. The present study was supported by grants from the National Natural Science Foundation of China (nos. 30873041 , 81101928 and 81025015$)$.

\section{References}

1. Jemal A, Bray F, Center MM, Ferlay J, Ward E and Forman D: Global cancer statistics. CA Cancer J Clin 61: 69-90, 2011.

2. Chow WH, Dong LM and Devesa SS: Epidemiology and risk factors for kidney cancer. Nat Rev Urol 7: 245-257, 2010.

3. Southard EB, Roff A, Fortugno T, et al: Lead, calcium uptake, and related genetic variants in association with renal cell carcinoma risk in a cohort of male Finnish smokers. Cancer Epidemiol Biomarkers Prev 21: 191-201, 2012.

4. Wang G, Hou J, Ma L, et al: Risk factor for clear cell renal cell carcinoma in Chinese population: a case-control study. Cancer Epidemiol 36: 177-182, 2012.

5. Moore LE, Brennan P, Karami S, et al: Glutathione S-transferase polymorphisms, cruciferous vegetable intake and cancer risk in the Central and Eastern European Kidney Cancer Study. Carcinogenesis 28: 1960-1964, 2007.

6. Moore LE, Boffetta P, Karami S, et al: Occupational trichloroethylene exposure and renal carcinoma risk: evidence of genetic susceptibility by reductive metabolism gene variants. Cancer Res 70: 6527-6536, 2010.
7. Bruyère F, Hovens CM, Marson MN, et al: VEGF polymorphisms are associated with an increasing risk of developing renal cell carcinoma. J Urol 184: 1273-1278, 2010.

8. Hirata H, Hinoda Y, Nakajima K, et al: Wnt antagonist gene polymorphisms and renal cancer. Cancer 115: 4488-4503, 2009.

9. Andreotti G, Boffetta P, Rosenberg PS, et al: Variants in blood pressure genes and the risk of renal cell carcinoma. Carcinogenesis 31: 614-620, 2010.

10. Longuemaux S, Deloménie C, Gallou C, et al: Candidate genetic modifiers of individual susceptibility to renal cell carcinoma: a study of polymorphic human xenobiotic-metabolizing enzymes. Cancer Res 59: 2903-2908, 1999.

11. Aben KK, Luth TK, Janssen-Heijnen ML, Mulders PF, Kiemeney LA and van Spronsen DJ: No improvement in renal cell carcinoma survival: a population-based study in the Netherlands. Eur J Cancer 44: 1701-1709, 2008.

12. Sun M, Shariat SF, Cheng C, et al: Prognostic factors and predictive models in renal cell carcinoma: a contemporary review. Eur Urol 60: 644-661, 2011.

13. Lughezzani G, Karakiewicz PI, Bigot P, et al: The prognostic value of erythrocyte polyamines in the preoperative evaluation of patients with renal cell carcinoma. Eur J Cancer 46: 1927-1935, 2010.

14. Doberstein K, Wieland A, Lee SB, et al: L1-CAM expression in ccRCC correlates with shorter patients survival times and confers chemoresistance in renal cell carcinoma cells. Carcinogenesis 32: 262-270, 2011.

15. Kojima T, Shimazui T, Horie R, et al: FOXO1 and TCF7L2 genes involved in metastasis and poor prognosis in clear cell renal cell carcinoma. Genes Chromosomes Cancer 49: 379-389, 2010.

16. Li X, Tan X, Yu Y, et al: D9S168 microsatellite alteration predicts a poor prognosis in patients with clear cell renal cell carcinoma and correlates with the down-regulation of protein tyrosine phosphatase receptor delta. Cancer 117: 4201-4211, 2011.

17. Kleinrath T, Gassner C, Lackner P, Thurnher M and Ramoner R: Interleukin-4 promoter polymorphisms: a genetic prognostic factor for survival in metastatic renal cell carcinoma. J Clin Oncol 25: 845-851, 2007.

18. Hirata H, Hinoda Y, Kikuno N, et al: MDM2 SNP309 polymorphism as risk factor for susceptibility and poor prognosis in renal cell carcinoma. Clin Cancer Res 13: 4123-4129, 2007.

19. Lin J, Horikawa Y, Tamboli P, Clague J, Wood CG and Wu X: Genetic variations in microRNA-related genes are associated with survival and recurrence in patients with renal cell carcinoma. Carcinogenesis 31: 1805-1812, 2010.

20. Purdue MP, Johansson M, Zelenika D, et al: Genome-wide association study of renal cell carcinoma identifies two susceptibility loci on 2p21 and 11q13.3. Nat Genet 43: 60-65, 2011.

21. Schödel J, Bardella C, Sciesielski LK, et al: Common genetic variants at the 11q13.3 renal cancer susceptibility locus influence binding of HIF to an enhancer of cyclin D1 expression. Nat Genet 44: 420-425, 2012.

22. Chang W, Ma L, Lin L, et al: Identification of novel hub genes associated with liver metastasis of gastric cancer. Int J Cancer 125: 2844-2853, 2009.

23. Wu X, Scelo G, Purdue MP, et al: A genome-wide association study identifies a novel susceptibility locus for renal cell carcinoma on 12p11.23. Hum Mol Genet 21: 456-462, 2012.

24. Han SS, Yeager M, Moore LE, et al: The chromosome 2p21 region harbors a complex genetic architecture for association with risk for renal cell carcinoma. Hum Mol Genet 21: 1190-1200, 2012.

25. Cao Q, Qin C, Ju X, et al: Chromosome 11q13.3 variant modifies renal cell cancer risk in a Chinese population. Mutagenesis 27: 345-350, 2012.

26. Hedberg Y, Davoodi E, Roos G, Ljungberg B and Landberg G: Cyclin-D1 expression in human renal-cell carcinoma. Int J Cancer 84: 268-272, 1999.

27. Phuoc NB, Ehara H, Gotoh T, et al: Immunohistochemical analysis with multiple antibodies in search of prognostic markers for clear cell renal cell carcinoma. Urology 69: 843-848, 2007.

28. Allory Y, Matsuoka Y, Bazille C, Christensen EI, Ronco P and Debiec H: The L1 cell adhesion molecule is induced in renal cancer cells and correlates with metastasis in clear cell carcinomas. Clin Cancer Res 11: 1190-1197, 2005.

29. Migita T, Oda Y, Naito S and Tsuneyoshi M: Low expression of p27(Kip1) is associated with tumor size and poor prognosis in patients with renal cell carcinoma. Cancer 94: 973-979, 2002.

30. Bilim V, Kawasaki T, Katagiri A, Wakatsuki S, Takahashi K and Tomita Y: Altered expression of beta-catenin in renal cell cancer and transitional cell cancer with the absence of beta-catenin gene mutations. Clin Cancer Res 6: 460-466, 2000. 